\title{
ESTRATEGIAS DE AFRONTAMIENTO: EFECTOS EN EL BIENESTAR PSICOLÓGICO DE UNIVERSITARIOS DURANTE LA PANDEMIA DEL COVID-19
}

\section{Coping Strategies: Their Effects on University Students' Psychological Well-being During the COVID-19 Pandemic}

\section{Iris Bello Castillo}

Profesora de la Universidad Iberoamericana (UNIBE),

República Dominicana.

ORCID: 0000-0002-3791-2850

Correo-e: i.bello@prof.unibe.e du.do

Anny Lisbeth Martínez Camilo

Estudiante (UNIBE). ORCID: 0000-0002-0541-2165

Correo-e: martinez23@est.unibe.edu.do

\author{
Paulette Marie Peterson Elías \\ Estudiante (UNIBE). ORCID: 0000-0002-7886-3178 \\ Correo-e: ppeterson@est.unibe.edu.do \\ Laura V. Sánchez-Vincitore \\ Profesora (UNIBE). ORCID: 0000-0002-6343-1217 \\ Correo-e: 1.sanchez1@prof.unibe.edu.do
}

\section{Recibido: 21/7/2021 • Aprobado: 29/10/2021}

Cómo citar: Bello-Castillo, I., Martínez Camilo, A., Peterson Elías, P. M., \& Sánchez-Vincitore, L. V. (2021). Estrategias de Afrontamiento: efectos en el bienestar psicológico de universitarios durante la pandemia del COVID-19. Ciencia y Sociedad, 46(4), 31-48. Doi: https://doi.org/10.22206/cys.2021.v46i4.pp31-48

\section{Resumen}

Este estudio exploró la influencia de las estrategias de afrontamiento en el bienestar psicológico de estudiantes universitarios dominicanos durante la pandemia del COVID-19. Participaron 135 estudiantes de una universidad privada, entre 18 y 25 años, completando una encuesta virtual con la Escala de Bienestar Psicológico (EBP) y la escala de Orientación hacia el Afrontamiento de Problemas Experimentados (COPE-48). Seis regresiones múltiples con selección forward crearon modelos de predicción de cada dimensión del bienestar psicológico con las estrategias de afrontamiento como variables independientes. Los modelos explicaron del $23 \%$ al $40 \%$ de la varianza del bienestar psicológico. Un análisis factorial clasificó las estrategias de afrontamiento en: (1) positivas, ejerciendo una influencia positiva en el bienestar; y (2) negativas, ejerciendo una influencia negativa en el bienestar. Las estrategias positivas significativas fueron: (1) aceptación y crecimiento personal, y (2) búsqueda de apoyo social. Las estrategias negativas significativas fueron: (1) consumo de alcohol o drogas, (2) centrarse en las emociones y desahogarse, (3) negación, y (4) refrenar el afrontamiento. Las medidas de distanciamiento y la desconfianza en autoridades de salud durante

\begin{abstract}
This study aimed to determine which coping strategies influence psychological well-being of university students in the Dominican Republic during the COVID-19 pandemic. Participants from a private university in Santo Domingo between 18-25 participated $(\mathrm{n}=135)$. They completed an online survey with a sociodemographic section, the Psychological Well-being Scale (EBP), and the Coping Orientation to Problems Experienced scale (COPE-48). Six multiple regression analyses with forward selection were conducted to create prediction models for each dimension of psychological well-being with coping strategies' dimensions as independent variables. The models explained 23\% to $40 \%$ of the psychological well-being variance. A factor analysis classified the coping strategies as (1) positive, exerting a positive influence on well-being, and (2) negative, exerting a negative effect on well-being. The models' positive strategies were: (1) acceptance and personal growth and (2) search for social support. The model's negative strategies were: (1) alcohol or drug use, (2) focus on emotions and vent, (3) denial, and (4) restrain coping. Physical distancing measures and mistrust of health authorities during the pandemic make it difficult to engage in positive coping
\end{abstract}


la pandemia dificultan usar estrategias de afrontamiento positivas, favoreciendo el uso de estrategias negativas. Esto aumenta el riesgo de desarrollar sintomatologías clínicamente relevantes.

Palabras clave: Bienestar psicológico; afrontamiento; COVID-19; República Dominicana

\section{Introducción}

El COVID-19 es una enfermedad respiratoria infecciosa producida por el virus SARS-CoV-2 (Wu et al., 2020). Debido a la letalidad del virus, su forma y velocidad de transmisión (Dong et al., 2020), la Organización Mundial de la Salud (2020) recomienda a las personas prevenir el contagio a través del distanciamiento físico, el uso de mascarillas y el lavado frecuente de manos. Dependiendo de la capacidad de atención de los sistemas de salud de algunos países, sus líderes han declarado estados de emergencia, cerrado fronteras, ordenado toques de queda, y cerrado lugares de conglomeración de personas (Brauner et al., 2020).

El temor a contraer o propagar el virus (Tran et al., 2020), el malestar producido por las medidas para controlar su propagación, la inseguridad financiera y la incertidumbre sobre el futuro, han hecho del COVID-19 un potencial detonante de una crisis de salud mental (Van Bavel et al., 2020). Durante la pandemia, varios estudios han encontrado que las personas presentan niveles altos de ansiedad (Husky et al., 2020; Hyland et al., 2020), depresión (Rodríguez-Hidalgo et al., 2020; Salari et al., 2020), malestar psicológico (Daly \& Robinson, 2020; Lahav, 2020), y un descenso en la calidad de vida (Schiavi et al., 2020; Tran et al., 2020).

Si bien es cierto que las personas jóvenes y saludables no tienen altos riesgos de complicaciones por COVID-19 (Noor \& Islam, 2020; Williamson et al., 2020), la pandemia ha afectado a los estudiantes universitarios de una manera particular strategies, allowing people to more easily engage in strategies that negatively affect psychological well-being, increasing the risk of developing clinically relevant symptoms.

Keywords: Psychological Well-being; Coping; COVID-19; Dominican Republic

(Chen et al., 2020; Islam et al., 2020; Morote-Jayacc et al., 2020; Wang et al., 2020). A nivel de educación superior, las clases universitarias presenciales fueron suspendidas y los estudiantes tuvieron que adaptarse al aprendizaje virtual, usando plataformas para las que el sistema educativo aún no estaba preparado (Gianni, 2020). Gianni (2020) reporta que los estudiantes más vulnerables tienen nuevas cargas financieras, y que aquellos que se gradúan durante la pandemia tienen un mercado laboral incierto, lo que contribuye a un malestar general. En un estudio con participantes brasileños se encontró que estos presentaron niveles mayores de depresión en la pandemia cuando eran de posiciones socieconómicas más bajas, asistían a universidades públicas, no practicaban ninguna religión y estaban desempleados (Patias et al., 2021).

Los jóvenes universitarios tienen características que les hacen vulnerables a los efectos del distanciamiento físico y de las demás restricciones (Cao et al., 2020). Por ejemplo, en la adultez emergente se toman decisiones sobre la formación académica, el ámbito laboral, relaciones sociales y de pareja, que son determinantes para su desarrollo y para el bienestar general Wood et al. (2018), y que son afectados durante la pandemia del COVID-19.

En Estados Unidos, un estudio encontró que los estudiantes universitarios mostraban preocupación por su futuro académico, altos niveles de etrés, y dificultades de ajuste ante los cambios provocados por el COVID-19 (Clabaugh et al., 2021). Estos resultados fueron particularmente marcados en minorías, tales como mujeres y personas afrodescendientes. 


\section{Estrategias de Afrontamiento: efectos en el bienestar psicológico de universitarios durante la pandemia del COVID-19}

El bienestar se refiere al funcionamiento psicológico óptimo y experiencias óptimas de las personas (Ryan $\&$ Deci, 2001). El constructo ha sido estudiado desde dos abordajes filosóficos: (1) hedonismo, caracterizado por la búsqueda del placer (bienestar subjetivo); y (2) eudamonia, caracterizada por la búsqueda de la autorrealización (bienestar psicológico). El bienestar subjetivo, también conocido como felicidad, está conformado por las dimensiones: satisfacción con la vida; presencia de afecto positivo, refiriéndose a un estado de ánimo placentero; y ausencia de afecto negativo, es decir, ausencia de malestar (Ryan \& Deci, 2001). El bienestar psicológico se refiere al funcionamiento óptimo, en el que la persona se evalúa a sí misma y sus experiencias en seis dimensiones: autoaceptación, relaciones positivas, autonomía, dominio del ambiente, propósito de la vida y crecimiento personal (Ryff, 2014; Ryff \& Singer, 1996). Las valoraciones altas en estas dimensiones se relacionan con una mayor satisfacción con la vida. Joshanloo $(2016,2019)$ encontró que, aunque el bienestar psicológico y el bienestar subjetivo son conceptos empíricamente distintos, estos se correlacionan.

Anglim y Horwood (2021) hallaron que una muestra de estudiantes universitarios encuestados durante la pandemia obtuvo puntuaciones significativamente menores en bienestar psicológico y bienestar subjetivo, comparado con una muestra de estudiantes universitarios antes de la pandemia. Estas diferencias fueron evidentes para las dimensiones de bienestar psicológico de autoaceptación, relaciones positivas, dominio del entorno y propósito de la vida; y para las dimensiones de bienestar subjetivo en la reducción de la satisfacción con la vida y afecto positivo, y un aumento del afecto negativo.

La literatura reporta que algunos factores, tanto externos como internos, amortiguan el efecto negativo de la pandemia en el bienestar y la salud mental. Entre los factores externos están las medidas de confinamiento que incluyen apoyo económico por parte de los gobiernos (Foa et al., 2020); también conocer suficiente información sobre COVID-19, especialmente cuando las personas tienen altos niveles de afecto positivo y bajos niveles de afecto negativo (Blasco-Belled et al., 2020). Entre los factores internos están las estrategias de afrontamiento (Dawson \& Golijani-Moghaddam, 2020; MacIntyre et al., 2020; Rogowska et al., 2020; Zacher \& Rudolph, 2021).

\section{Estrategias de afrontamiento}

Las estrategias de afrontamiento se definen como respuestas voluntarias o automáticas al estrés, ya sean cognitivas, emocionales, o conductuales (Stanisławski, 2019). Según la forma en la que la persona se enfrenta a la situación estresante, el afrontamiento puede ser manifestado acercándose al problema - afrontamiento de acercamiento - o distanciándose del problema afrontamiento evitativo- (Gol \& Cook, 2004; Roth \& Cohen, 1986). Según las expectativas de la respuesta al estrés, el afrontamiento puede llevar a solucionar activamente el problema —afrontamiento orientado al problema - o a regular la respuesta emocional que causa el problema -afrontamiento orientado a la emoción— (Folkman \& Lazarus, 1980). Y según el nivel de apoyo necesario para enfrentar el problema, el afrontamiento puede llevarse a cabo individualmente - afrontamiento autosuficiente - o grupalmente afrontamiento de apoyo social- (Litman, 2006). Estas categorías no son mutuamente excluyentes, ya que varias estrategias de afrontamiento pueden pertenecer a diferentes categorizaciones de manera simultánea según la función que cumplan y el contexto en el que se den (Stanisławski, 2019). Litman (2006) indica que generalmente el afrontamiento autosuficiente $(v$. gr. afrontamiento activo del problema o aceptación) y de apoyo social ( $v . g r$ búsqueda de apoyo emocional en otras personas) forman parte del constructo de afrontamiento de acercamiento, que a su vez está asociado al afecto positivo; mientras que el afrontamiento evitativo ( $v$. gr. la negación y el uso de alcohol y otras sustancias) es un factor separado, y está asociado al afecto 
negativo. Las tres estrategias de afrontamiento - autosuficiente, de apoyo social y de evitación — pueden ser orientadas tanto al problema como a la emoción.

En un estudio con participantes trabajadores de Alemania, realizado durante la pandemia del COVID-19, Zacher y Rudolph (2020) encontraron relaciones estadísticamente significativas entre estrategias de afrontamiento y el bienestar subjetivo. Las estrategias de afrontamiento orientadas al problema, las orientadas a emociones y las de apoyo social se relacionaron de manera positiva con las dimensiones de bienestar subjetivo de satisfacción de la vida y de afecto positivo. De igual forma, las estrategias de afrontamiento evitativo se relacionaron de manera positiva con la dimensión del bienestar subjetivo de afecto negativo, y de manera negativa con las estrategias de apoyo social. Resultados similares fueron encontrados en una muestra de profesores de idiomas en Europa, América del Norte, América del Sur y Oriente Medio, en la que las estrategias de afrontamiento orientadas al problema correlacionaron de manera positiva con el bienestar, mientras que las estrategias de afrontamiento evitativo correlacionaron de manera negativa con el bienestar (MacIntyre et al., 2020). Rogowska et al. (2020) encontraron correlaciones positivas entre la satisfacción con la vida y las estrategias de afrontamiento orientadas al problema, y a diferencia de otros estudios, correlaciones negativas entre la satisfacción con la vida y las estrategias de afrontamiento orientadas a la emoción en estudiantes polacos. Un estudio con participantes adultos de Reino Unido encontró una relación significativa entre factores sociodemográficos y psicolosciales con el tipo de estrategia de afrontamiento que emplea una persona durante la pandemia (Fluharty \& Fancourt, 2021). Adicionalmente, dicho estudio encontró un patrón específico de uso de estrategias de afrontamiento relacionadas a las adversidades específicas producidas por COVID-19, en especial, cuando atentan contra las finanzas personales.
El afrontamiento durante la pandemia ha sido explorado en estudiantes universitarios. Una investigación con estudiantes universitarios de Turquía encontró un efecto amortiguador del optimismo y la esperanza en la relación entre el estrés por la pandemia del COVID-19 y el bienestar subjetivo (Genç \& Arslan, 2021). Asimismo, en una investigación con estudiantes universitarios de la Florida, se encontró una relación significativa entre el uso de estrategias de afrontamiento y bienestar emocional (Jacobs et al., 2021). Los estudiantes que emplean estrategias de afrontamiento positivas, especialmente encontrar el beneficio a la situación de crisis, generalmente presentan menos discurso de miedo, ansiedad y estrés (August $\&$ Dapkewicz, 2021). De igual forma, una investigación con estudiantes filipinos encontró una correlación positiva entre las estrategias de afrotamiento de acercamiento (positivas) y el bienestar en tres dimensiones (bienestar académico, social y mental); mientras que encontró una correlación negativa entre las estrategias de afrontamiento evitativas (negativas) y el bienestar académico (Valladolid, 2021).

Si bien los efectos positivos de algunas estrategias de afrontamiento en el bienestar durante la pandemia han sido explorados en países post industrializados, en el momento de esta publicación, existe poca evidencia de su exploración en América Latina y el Caribe. Un estudio mexicano encontró que los participantes tenían consumo emocional de alimentos como posible mecanismo de afrontamiento durante la pandemia, específicamente cuando presentaban altos niveles de estrés, participaban de conductas negativas, tenían niveles altos de ansiedad, convivían con una persona diagnosticada con COVID-19 y género femenino (Carillo et al., 2021). De igual forma, el estudio encontró que el apoyo social funcionaba como variable protectora.

En la República Dominicana se detectó el primer caso de COVID-19 a principios de marzo del 2020 (Rathe, 2020). A enero del 2021 se reportaron 201,145 casos confirmados, 146,491 casos recu- 


\section{Estrategias de Afrontamiento: efectos en el bienestar psicológico de universitarios durante la pandemia del COVID-19}

perados y 2,496 personas fallecidas (Ministerio de Salud Pública de la República Dominicana, 2021). Mencía-Ripley et al. (2021) reportan que la población general ha experimentado malestar psicológico durante la pandemia del COVID-19, situación exacerbada por la percepción negativa del personal de salud y falta de confianza a las autoridades.

Teniendo en cuenta que el bienestar psicológico de jóvenes universitarios a nivel mundial está en riesgo de ser afectado por los efectos de las medidas de prevención COVID-19, es necesario estudiar los mecanismos que pueden contrarrestar este efecto negativo. El propósito de este estudio fue conocer la influencia de las estrategias de afrontamiento en las distintas dimensiones del bienestar psicológico en jóvenes universitarios de la República Dominicana durante la pandemia del COVID-19. El trabajo presenta las siguientes preguntas de investigación: (1) ¿Las estrategias de afrontamiento predicen el bienestar psicológico? Hipotetizamos que las estrategias de afrontamiento predicen significativamente los distintos niveles de bienestar psicológico; (2) ¿Cuáles son las estrategias de afrontamiento que mejor predicen las diferentes dimensiones del bienestar psicológico? Hipotetizamos que las estrategias de afrontamiento relacionadas a la socialización, que ha sido afectada durante la pandemia del COVID19, predecirá en mayor medida el bienestar; (3) ¿Las estrategias de afrontamiento evaluadas se clasifican en positivas y negativas, como lo proponen Gol y Cook (2004)? Hipotetizamos que el constructo de afrontamiento se clasificará en dos factores: positivo y negativo; (4) ¿De qué manera predicen las estrategias de afrontamiento positivas y negativas el bienestar psicológico? Hipotetizamos que los coeficientes de regresión de las estrategias de afrontamiento positivas (ya sean de autosuficiencia o apoyo social) predicen un aumento en los niveles de bienestar psicológico. Asimismo, hipotetizamos que los coeficientes de regresión de las estrategias de afrontamiento negativas (evitativas) predicen una disminución en los niveles de bienestar psicológico.

\section{Métodos}

\section{Diseño}

La presente investigación tiene un diseño no experimental transversal.

\section{Participantes}

Se reportan datos de 135 participantes $(74.07 \%$ mujeres) jóvenes universitarios de 18 a 25 años $(M=$ 20.47, $D T=1.53)$, residentes de la ciudad de Santo Domingo, República Dominicana, que vivieron la cuarentena domiciliaria en dicha ciudad. Los participantes reportan un promedio de posición socioeconómica media-alta.

Para determinar el tamaño de muestra, se realizó un análisis de potencia para una regresión múltiple de dos colas, con un tamaño de efecto moderado $\left(f^{2}=.15\right)$, alfa de .05 , una potencia de .95 y cuatro predictores, para un tamaño de muestra de 89 participantes.

\section{Instrumentos}

Para conocer las características sociodemográficas de la muestra, administramos el Cuestionario sociodemográfico creado por las autoras. Este cuestionario indagaba información de edad, sexo, estado civil, grado académico, ocupación, lugar de residencia, lugar dónde pasó la cuarentena por COVID-19. Este cuestionario incluyó la escala de posición socioeconómica percibida de McArthur, que solicita a los participantes ubicarse en una escalera de 10 peldaños. Se les explica que en el primer peldaño se encuentran las personas a quienes peor les va en la vida (en cuanto a trabajo y educación), y que en el décimo peldaño se encuentran las personas a quienes mejor les va en la vida. Los participantes escogen el peldaño en el que se perciben.

Para medir la variable de bienestar psicológico se utilizó la adaptación española de la Escala de Bienestar Psicológico de Díaz et al. (2006) de las 
versiones en inglés de Ryff (1989) y van Dierendonck et al. (2008). Este instrumento mide el crecimiento personal y funcionamiento adecuado de las personas a partir del desarrollo de ciertas habilidades. La versión adaptada en español a utilizar consta de 39 ítems y 6 subescalas utilizando una escala de tipo Likert. Cada ítem solicita al participante que indique qué tanto se identifica con los enunciados en puntuaciones que van desde el valor de 1 (no se identifica nada) hasta 6 (extremadamente identificado). Las subescalas del instrumento son: autoaceptación, relaciones positivas, dominio del entorno y propósito en la vida, autonomía y crecimiento personal. La subescala de autoaceptación mide las actitudes sobre sí mismo y la percepción de las cualidades personales, incluyendo sus fortalezas y limitaciones. La subescala de relaciones positivas mide la calidad de los vínculos con los demás, empatía, afecto e intimidad. La subescala de autonomía informa sobre la habilidad de expresar los propios ideales y poder autorregular la propia conducta con independencia. La subescala del dominio del entorno se define como la capacidad de una persona para identificar los lugares donde le sea posible satisfacer sus propias necesidades y deseos. La subescala de propósito en la vida mide la tendencia de la persona de encontrar sentido a la vida al proponerse metas. La subescala de crecimiento personal mide la actitud de estar en constante desarrollo y fortalecimiento de las propias capacidades para cumplir los objetivos planteados a lo largo de la vida. El instrumento obtuvo una consistencia interna de aceptable a muy buena, evidenciada por puntuaciones de alfa de Cronbach que oscilaban entre .66 y .85 para las subescalas.

Para medir las variables de estrategias de afrontamiento se utilizó el Cuestionario de Orientación para Afrontar Problemas Experimentados (COPE-48, por sus siglas en inglés) creada por Carver (1997) y Carver et al. (1989), y adaptada al español por Martínez-Ortega et al. (2018) y Martínez Ortega et al. (2016). Este instrumento mide la frecuencia de uso de estrategias de afrontamiento. La versión espańola consta de 48 ítems y 9 subescalas con una escala tipo Likert. Cada ítem solicita a los participantes indicar la frecuencia con la que emplea las estrategias de afrontamiento presentadas, con respuestas desde el valor 1 (no lo hago nunca) hasta el valor 4 (lo hago muchas veces). Este instrumento cuenta de las siguientes subescalas: afrontamiento activo del problema, consumo de alcohol o drogas, centrarse en las emociones y desahogarse, búsqueda de apoyo social, humor, religión, negación, refrenar el afrontamiento, y aceptación y crecimiento. La subescala de afrontamiento activo del problema mide qué tanto la persona da pasos en concreto y planifica cómo va a resolver alguna situación estresante. La subescala de consumo de alcohol o drogas mide qué tanto la persona consume alcohol o drogas para sentirse bien o aliviar el malestar causado por una situación estresante. La subescala de centrarse en las emociones y desahogarse mide qué tanto la persona se enfoca en la situación estresante quejándose de la misma. La subescala de búsqueda de apoyo social mide qué tanto la persona solicita asistencia, ayuda o información tanto para resolver la situación estresante, como buscando apoyo moral y empatía. La subescala de humor mide qué tanto la persona tiende a burlarse y reírse de las situaciones estresantes. La subescala religión que mide la tendencia de dirigirse hacia la espiritualidad o religión en momentos de estrés. La subescala de negación mide la frecuencia con la que la persona desestima el estresor negando su existencia. La subescala de refrenar el afrontamiento mide qué tanto la persona actúa de manera pasiva ante la situación estresante, en aras de esperar a que sea un momento adecuado para afrontar el estresor. La subescala de aceptación y crecimiento mide qué tanto la persona es capaz de entender y aceptar la situación estresante y regular sus emociones alrededor de la misma. El instrumento obtuvo una consistencia interna de buena a muy buena, evidenciada por puntuaciones de alfa de Cronbach que oscilaban entre .70 y .97 para las subescalas. 


\section{Estrategias de Afrontamiento: efectos en el bienestar psicológico de universitarios durante la pandemia del COVID-19}

\section{Procedimiento}

Los participantes fueron contactados a través de las redes sociales. En esta encuesta virtual se presentó una sección de consentimiento informado, donde los participantes conocieron los beneficios y riesgos de su participación en la investigación, y tuvieron una opción de especificar si estaban de acuerdo o no con formar parte de la investigación. De igual forma, se les informó que su participación era completamente anónima. En caso de afirmar su participación, se les facilitó un espacio donde firmaron con sus iniciales.

\section{Consideraciones éticas}

El presente estudio contó con la aprobación del Comité de ética de la Universidad Iberoamericanan (UNIBE), siguiendo las declaraciones universales de los principios éticos de la psicología.

\section{Resultados}

Para conocer las características de las variables del estudio, la tabla 1 presenta las estadísticas descriptivas de las puntuaciones en cada subescala de cada instrumento de evaluación.

\section{Tabla 1}

\section{Estadisticas descriptivas de las variables del estudio}

\begin{tabular}{|c|c|c|c|c|c|}
\hline Variables & $\mathbf{N}$ & $\mathbf{M}$ & DT & Min & Max \\
\hline \multicolumn{6}{|l|}{ Bienestar psicológico } \\
\hline Autoaceptación & 135 & 4.43 & 0.96 & 1.17 & 6 \\
\hline Relaciones positivas & 135 & 4.43 & 0.95 & 2 & 6 \\
\hline Autonomía & 135 & 4.02 & 0.80 & 2.5 & 6 \\
\hline Dominio del ambiente & 135 & 4.40 & 0.86 & 1.33 & 6 \\
\hline Propósito de la vida & 135 & 4.77 & 0.88 & 2 & 6 \\
\hline Crecimiento personal & 135 & 4.54 & 1.01 & 1 & 6 \\
\hline \multicolumn{6}{|l|}{ Estrategias de afrontamiento } \\
\hline $\begin{array}{l}\text { Afrontamiento activo del } \\
\text { problema }\end{array}$ & 135 & 2.80 & 0.63 & 1.22 & 4 \\
\hline Consumo de alcohol o drogas & 135 & 1.53 & 0.89 & 1 & 4 \\
\hline $\begin{array}{l}\text { Centrarse en las emociones y } \\
\text { desahogarse }\end{array}$ & 135 & 2.48 & 0.77 & 1 & 4 \\
\hline
\end{tabular}

\begin{tabular}{lccccc}
\hline \multicolumn{1}{c}{ Variables } & N & M & DT & Min & Max \\
\hline Búsqueda de apoyo social & 135 & 2.99 & 0.77 & 1 & 4 \\
Humor & 135 & 2.54 & 0.95 & 1 & 4 \\
Religión & 135 & 2.67 & 1.00 & 1 & 4 \\
Negación & 135 & 1.59 & 0.86 & 1 & 4 \\
Refrenar el afrontamiento & 135 & 2.50 & 0.67 & 1 & 4 \\
$\begin{array}{l}\text { Aceptación y crecimiento } \\
\text { personal }\end{array}$ & 135 & 3.30 & 0.56 & 1.43 & 4 \\
\hline
\end{tabular}

Nota: $\mathrm{N}$ = tamaño muestral; $\mathrm{M}=$ media; $\mathrm{DT}$ = Desviación típica; Min $=$ Valor mínimo; Max $=$ Valor máximo

Para contestar la primera y segunda preguntas de investigación, se realizaron análisis de regresión múltiple con método forward de selección de predictores. Estas preguntas abordan si las estrategias de afrontamiento predicen el bienestar psicológico, y cuáles estrategias predicen mejor el bienestar psicológico. El método forward crea tantos modelos predictivos como predictores aporten al mismo, empezando por el predictor que tenga el mayor coeficiente de regresión, y terminando por el predictor de menor coeficiente de regresión, pero cuya influencia en el modelo es significativa. Los predictores que no aportan al modelo son excluidos. Se realizaron seis análisis de regresión para predecir las variables dependientes (dimensiones del bienestar psicológico: autoaceptación, relaciones positivas, autonomía, dominio del entorno, propósito de la vida y crecimiento personal), con las variables independientes (estrategias de afrontamiento: afrontamiento activo del problema, consumo de alcohol o drogas, centrarse en las emociones y desahogarse, búsqueda de apoyo social, humor, religión, negación, aceptación y crecimiento personal).

Para la variable dependiente de autoaceptación (véase tabla 2), el modelo de ajuste más adecuado identificó cuatro predictores: aceptación y crecimiento personal $(\beta=.49 \mathrm{p}<.001)$, consumo de alcohol o drogas $(\beta=-.25, \mathrm{p}=.001)$, refrenar el afrontamiento $(\beta=-.21 \mathrm{p}=.008)$, y búsqueda de apoyo social $(\beta=.16, \mathrm{p}=.028)$. El modelo explicó el $40 \%$ de la varianza $\left(\mathrm{R}^{2}=.40, \mathrm{~F}(4,130)=21.40\right.$, $\mathrm{p}<.001)$. 


\section{Tabla 2}

Regresión múltiple con variable dependiente autoaceptación y variables independientes estrategias de afrontamiento

\begin{tabular}{|c|c|c|c|c|c|}
\hline & $B$ & $S E B$ & $\beta$ & $R^{2}$ & $F$ \\
\hline \multicolumn{6}{|l|}{ Autoaceptación } \\
\hline Paso 1 & & & & 0.26 & $47.75^{* * *}$ \\
\hline Aceptación y crecimiento personal & 0.88 & 0.13 & $0.51^{* * *}$ & & \\
\hline Paso 2 & & & & 0.35 & $35.76^{* * *}$ \\
\hline Aceptación y crecimiento personal & 0.84 & 0.12 & $0.49^{* * *}$ & & \\
\hline Consumo de alcohol o drogas & -0.32 & 0.08 & $-0.30^{* * *}$ & & \\
\hline Paso 3 & & & & 0.37 & $26.11^{* * *}$ \\
\hline Aceptación y crecimiento personal & 0.88 & 0.12 & $0.52^{* * *}$ & & \\
\hline Consumo de alcohol o drogas & -0.25 & 0.08 & $-0.23^{* *}$ & & \\
\hline Refrenar el afrontamiento & -0.24 & 0.11 & $-0.17^{*}$ & & \\
\hline Paso 4 & & & & 0.4 & $21.40^{* * *}$ \\
\hline Aceptación y crecimiento personal & 0.83 & 0.12 & $0.49^{* * *}$ & & \\
\hline Consumo de alcohol o drogas & -0.27 & 0.08 & $-0.25^{* *}$ & & \\
\hline Refrenar el afrontamiento & -0.30 & 0.11 & $-0.21^{* *}$ & & \\
\hline Búsqueda de apoyo social & 0.20 & 0.09 & $0.16^{*}$ & & \\
\hline
\end{tabular}

${ }^{*} \mathrm{p}<.05 .{ }^{* *} \mathrm{p}<.01{ }^{* * *} \mathrm{p}<.001$

Para la variable dependiente de relaciones positivas (véase tabla 3), el modelo de ajuste más adecuado identificó dos predictores: consumo de alcohol o drogas $(\beta=-.41, \mathrm{p}<.001)$, y aceptación y crecimiento personal $(\beta=.23, \mathrm{p}=.004)$. El modelo explicó el $23 \%$ de la varianza $\left(\mathrm{R}^{2}=.23\right.$, $\mathrm{F}(2,132)=19.64, \mathrm{p}<.001)$.

\section{Tabla 3}

Regresión múltiple con variable dependiente relaciones positivas y variables independientes estrategias de afrontamiento

\begin{tabular}{lccccc}
\hline & $B$ & $S E B$ & $\beta$ & $R^{2}$ & $F$ \\
\hline $\begin{array}{l}\text { Relaciones positivas } \\
\text { Paso 1 }\end{array}$ & & & & 0.18 & $28.89^{* * *}$ \\
$\quad$ Consumo de alcohol o drogas & -0.45 & 0.08 & $-0.42^{* * *}$ & & \\
Paso 2 & & & & 0.23 & $19.64^{* * *}$ \\
$\quad$ Consumo de alcohol o drogas & -0.43 & 0.08 & $-0.41^{* * *}$ & & \\
$\quad$ Aceptación y crecimiento personal & 0.38 & 0.13 & $0.23^{* *}$ & & \\
\hline
\end{tabular}

${ }^{*} \mathrm{p}<.05 .{ }^{* *} \mathrm{p}<.01 .^{* * *} \mathrm{p}<.001$

Para la variable dependiente autonomía (véase y aceptación y crecimiento personal $(\beta=.30, p$ tabla 4), el modelo de ajuste más adecuado identi- $\quad<.001)$. El modelo explicó el $23 \%$ de la varianza ficó dos predictores: negación $(\beta=-.33 \mathrm{p}<.001), \quad\left(\mathrm{R}^{2}=.23, \mathrm{~F}(2,132)=19.22, \mathrm{p}<.001\right)$. 


\section{Tabla 4}

Regresión múltiple con variable dependiente autonomía y variables independientes estrategias de afrontamiento

\begin{tabular}{|c|c|c|c|c|c|}
\hline & $B$ & $S E B$ & B & $R^{2}$ & $F$ \\
\hline \multicolumn{6}{|l|}{ Autonomía } \\
\hline Paso 1 & & & & 0.14 & $21.46^{* * *}$ \\
\hline Negación & -0.34 & 0.07 & $-.37^{* * *}$ & & \\
\hline Paso 2 & & & & .23 & $19.22^{* * *}$ \\
\hline Negación & -0.30 & 0.07 & $-.33^{* * *}$ & & \\
\hline Aceptación y crecimiento personal & -0.42 & 0.11 & $.30^{* * *}$ & & \\
\hline
\end{tabular}

${ }^{*} \mathrm{p}<.05 .{ }^{* *} \mathrm{p}<.01 .{ }^{* * *} \mathrm{p}<.001$

Para la variable dependiente dominio del ambiente (véase tabla 5), el modelo de ajuste más adecuado identificó dos predictores: aceptación y crecimiento $(\beta=.46, p<.001), y$ refrenar el afrontamiento $(\beta=-.21, \mathrm{p}=.006)$. El modelo explicó el $23 \%$ de la varianza $\left(\mathrm{R}^{2}=.23, \mathrm{~F}(2,132)=19.99, \mathrm{p}<.001\right)$.

\section{Tabla 5}

Regresión múltiple con variable dependiente dominio del ambiente y variables independientes estrategias de afrontamiento

$\begin{array}{lllll}B & S E B & \beta & R^{2} & F\end{array}$

\section{Dominio del ambiente}

\section{Paso 1}

Aceptación y crecimiento personal

Paso 2

Aceptación y crecimiento personal

Refrenar el afrontamiento
0.70

$-0.27$
0.19

$30.73^{* * *}$

$0.66 \quad 0.12 \quad .43^{* * *}$

.23

$19.99^{* * *}$

${ }^{*} \mathrm{p}<.05 .{ }^{* *} \mathrm{p}<.01 .{ }^{* * *} \mathrm{p}<.001$

Para la variable dependiente propósito de vida (véase tabla 6), el modelo de ajuste más adecuado identificó cuatro predictores: aceptación y crecimiento personal $(B=.41, \mathrm{p}<.001)$, refrenar el afrontamiento $(\beta=-.28, \mathrm{p}=.001)$, consumo de alcohol o drogas $(\beta=-.21, p=.008)$, y búsqueda de apoyo social $(\beta=.15, \mathrm{p}=.049)$. El modelo explicó el $33 \%$ de la varianza $\left(\mathrm{R}^{2}=.33, \mathrm{~F}(4,130)\right.$ $=15.97, \mathrm{p}<.001)$. La tabla 6 contiene todos los pasos del análisis. 


\section{Tabla 6}

Regresión múltiple con variable dependiente propósito de la vida y variables independientes estrategias de afrontamiento

\begin{tabular}{|c|c|c|c|c|c|}
\hline & $B$ & $S E B$ & $\beta$ & $R^{2}$ & $F$ \\
\hline \multicolumn{6}{|l|}{ Propósito de la vida } \\
\hline Paso 1 & & & & .18 & $28.28^{* * *}$ \\
\hline Aceptación y crecimiento personal & 0.66 & 0.12 & $.42^{* * *}$ & & \\
\hline Paso 2 & & & & .28 & $25.14^{* * *}$ \\
\hline Aceptación y crecimiento personal & 0.71 & 0.12 & $.46^{* * *}$ & & \\
\hline Refrenar el afrontamiento & -0.42 & 0.10 & $-0.32^{* * *}$ & & \\
\hline Paso 3 & & & & .31 & $19.53^{* * *}$ \\
\hline Aceptación y crecimiento personal & 0.68 & 0.12 & $.43^{* * *}$ & & \\
\hline Refrenar el afrontamiento & -0.32 & 0.11 & $-.24^{* *}$ & & \\
\hline Consumo de alcohol o drogas & -0.20 & 0.08 & $-.20^{*}$ & & \\
\hline Paso 4 & & & & .33 & $15.97^{* * *}$ \\
\hline Aceptación y crecimiento personal & 0.64 & 0.12 & $.41^{* * *}$ & & \\
\hline Refrenar el afrontamiento & -0.37 & 0.11 & $-.28^{* *}$ & & \\
\hline Consumo de alcohol o drogas & -0.21 & 0.08 & $-.21^{* *}$ & & \\
\hline Búsqueda de apoyo social & 0.17 & 0.09 & $.15^{*}$ & & \\
\hline
\end{tabular}

${ }^{*} \mathrm{p}<.05 .{ }^{* *} \mathrm{p}<.01 .{ }^{* * *} \mathrm{p}<.001$

Para la variable dependiente crecimiento personal (véase tabla 7), el modelo de ajuste más adecuado identificó tres predictores: aceptación y crecimiento personal $(\beta=.28, \mathrm{p}<.001)$, búsqueda de apoyo social $(\beta=-.36, p<.001)$ y centrarse en las emociones $y$ desahogarse $(\beta=-.25, \mathrm{p}=.005)$. El modelo explicó el $23 \%$ de la varianza $\left(\mathrm{R}^{2}=.23, \mathrm{~F}(3,131)=12.93\right.$, $\mathrm{p}<.001)$.

\section{Tabla 7}

Regresión múltiple con variable dependiente crecimiento personal y variables independientes estrategias de afrontamiento

\begin{tabular}{llllll}
\hline & $B$ & $S E B$ & $\beta$ & $R^{2}$ & $F$ \\
\hline $\begin{array}{l}\text { Crecimiento personal } \\
\text { Paso 1 }\end{array}$ & & & & & \\
$\quad$ Aceptación y crecimiento personal & 0.63 & 0.15 & $0.35^{* * *}$ & & $18.99^{* * *}$ \\
Paso 2 & & & & 0.18 & $14.46^{* * *}$ \\
$\quad$ Aceptación y crecimiento personal & 0.56 & 0.14 & $0.31^{* * *}$ & & \\
$\quad$ Búsqueda de apoyo social & 0.31 & 0.11 & $0.24^{* *}$ & & \\
Paso 3 & & & & 0.23 & $12.93^{* * *}$ \\
$\quad$ Aceptación y crecimiento personal & 0.51 & 0.14 & $0.28^{* * *}$ & & \\
$\quad$ Búsqueda de apoyo social & 0.47 & 0.12 & $0.36^{* * *}$ & & \\
\hline Centrarse en las emociones y desahogarse & -0.33 & 0.11 & $-0.25^{* *}$ & & \\
\hline
\end{tabular}

${ }^{*} \mathrm{p}<.05 .{ }^{* *} \mathrm{p}<.01 .{ }^{* * *} \mathrm{p}<.001$ 


\section{Estrategias de Afrontamiento: efectos en el bienestar psicológico de universitarios durante la pandemia del COVID-19}

Para contestar la tercera pregunta de investigación sobre la categorización de tipos de estrategias de afrontamiento, se realizó un análisis de componentes principales de las subescalas de estrategias de afrontamiento con rotación varimax, permitiendo extraer dos factores. Los resultados de la prueba de esfericidad de Bartlett indican que es apropiado usar un análisis factorial en estos datos, debido a la significancia general de las correlaciones en la matriz de correlaciones $\left(\mathrm{X}^{2}(36)=317.03, \mathrm{p}<.001\right)$. De igual forma, la medición de Kaiser-Meyer-Oklin indica que es aceptable proceder con el análisis $(\mathrm{KMO}=.70)$. El análisis organizó las subescalas en dos componentes: (1) Afrontamiento negativo, incluyendo consumo de alcohol o drogas, negación, centrarse en las emociones $\mathrm{y}$ desahogarse, refrenar el afrontamiento, y humor; (2) Afrontamiento positivo, incluyendo aceptación y crecimiento personal, búsqueda de apoyo social, religión y afrontamiento activo del problema. Ambos factores explican el $52.29 \%$ de la varianza. La tabla 8 contiene los valores de cada componente.

\section{Tabla 8}

Matriz de componentes rotados de estrategias de afrontamiento

\begin{tabular}{lcc}
\hline & $\mathbf{1}$ & $\mathbf{2}$ \\
\hline Consumo de alcohol o drogas & 0.82 & \\
Negación & 0.78 & \\
Centrarse en las emociones y desahogarse & 0.67 & \\
Refrenar el afrontamiento & 0.62 & \\
Humor & 0.53 & \\
Afrontamiento activo del problema & & 0.78 \\
Religión & & 0.77 \\
Búsqueda de apoyo social & & 0.71 \\
Aceptación y crecimiento personal & & 0.49 \\
\hline
\end{tabular}

Nota: el método de extracción fue análisis de componentes principales, rotación varimax con normalización de Kaiser.

Para contestar la cuarta pregunta de investigación sobre la manera en la que las estrategias de afrontamiento positivo y negativo predicen el bienestar psicológico, se creó una tabla de contingencia con las puntuaciones de beta estandarizadas en la intersección de las estrategias de afrontamiento y dimensiones del bienestar. La tabla 9 presenta un resumen de las puntuaciones de las betas estandarizadas de los modelos de predicción de cada dimensión de bien- estar psicológico organizados según tipo de estrategias de afrontamiento (positivas y negativas). Las estrategias positivas que contribuyeron a los modelos de predicción fueron la aceptación y crecimiento personal, y la búsqueda de apoyo social. Las estrategias negativas que contribuyeron a los modelos de predicción fueron el consumo de alcohol o drogas, refrenar el afrontamiento, centrarse en las emociones y desahogarse, y la negación. 


\section{Tabla 9}

Resumen de las puntuaciones de Beta de los predictores de los modelos de cada dimensión del bienestar psicológico

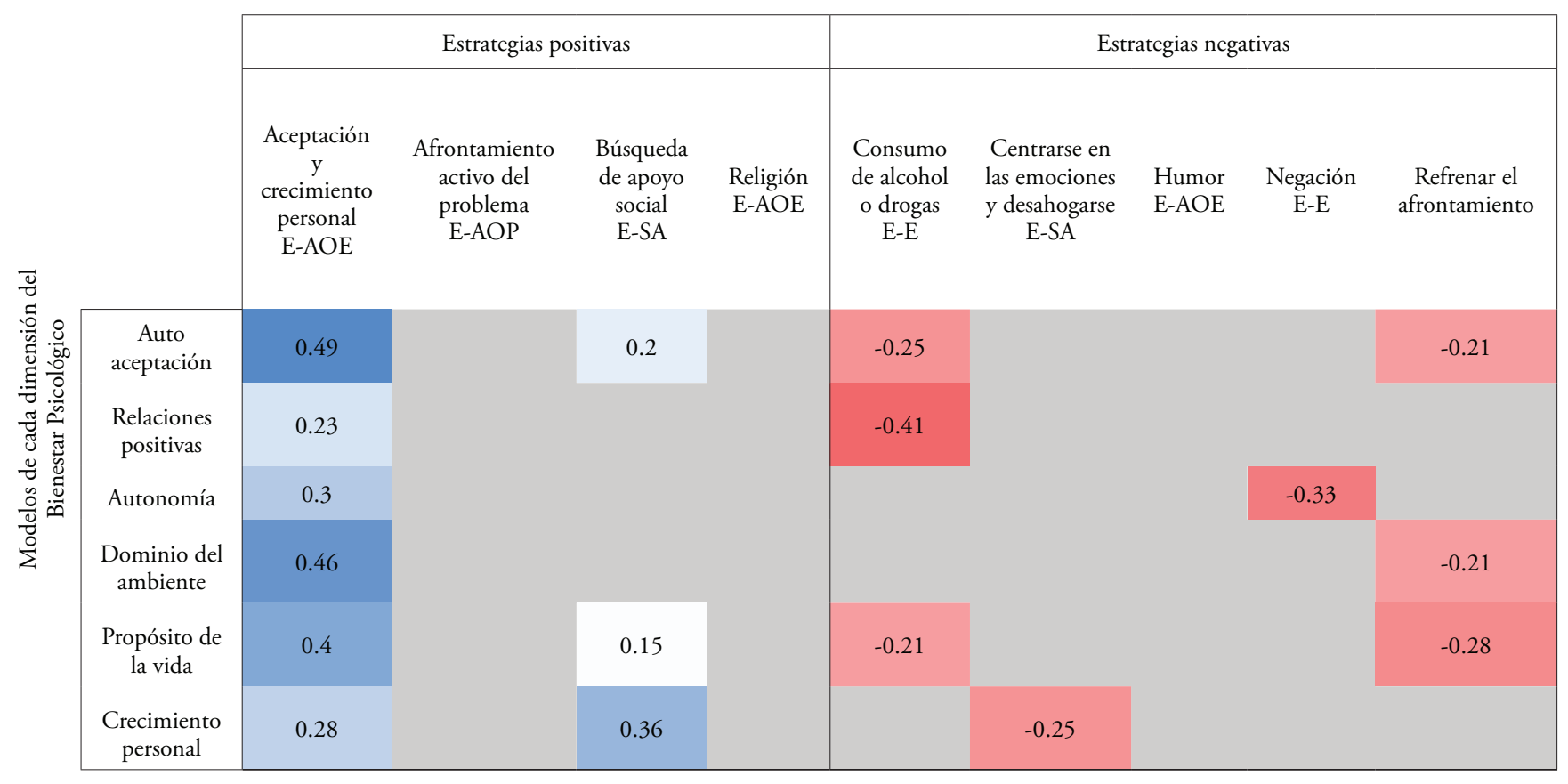

Betas de las variables independientes de estrategias de afrontamiento

Nota: los tonos rojos indican puntuaciones negativas, los tonos azules indican puntuaciones positivas, la intensidad de los colores indican la magnitud, el color gris indica que el predictor no entró en el modelo.

$\mathrm{E}-\mathrm{AOP}=$ estrategia autosuficiente orientada al problema; E-AOE = estrategia autosuficiente orientada a la emoción; E-SA = estrategia social de apoyo; $\mathrm{E}-\mathrm{E}=$ estrategia de evitación.

\section{Discusión}

El propósito de esta investigación consistió en conocer el efecto de distintas estrategias de afrontamiento en el bienestar psicológico de estudiantes universitarios dominicanos durante la pandemia del COVID-19.

La primera y segunda preguntas de investigación indagaban sobre las estrategias de afrontamiento como predictoras del bienestar psicológico. El estudio encontró que las estrategias de afrontamiento predicen el bienestar psicológico. Esto confirma el hallazgo de las investigaciones sobre el efecto del afrontamiento en distintas dimensiones del bienestar durante la pandemia (August \& Dapkewicz, 2021; Genç \& Arslan, 2021; Jacobs et al., 2021; Zacher \& Rudolph, 2021). En este estudio, las estrategias de mayor impacto al bienestar fueron la aceptación y crecimiento personal, búsqueda de apoyo social, consumo de alcohol o drogas, centrarse en las emociones y desahogarse, negación, y asumir una actitud pasiva ante el afrontamiento.

La tercera y cuarta preguntas de investigación buscaba categorizar las estrategias de afrontamiento y conocer su impacto en el bienestar. Según los resultados del análisis factorial de la macroestructura de las estrategias de afrontamiento, estas se categorizaron en positivas y negativas. Las estrategias positivas fueron: aceptación y crecimiento personal, afrontamiento activo del problema, búsqueda de apoyo social y religión. Las estrategias negativas fueron: consumo de alcohol o drogas, centrarse en las emociones y desahogarse, humor, negación 


\section{Estrategias de Afrontamiento: efectos en el bienestar psicológico de universitarios durante la pandemia del COVID-19}

y refrenar el afrontamiento. En el contexto de la pandemia del COVID-19, algunas de estas estrategias fueron relevantes, mientras que otras no. En referencia a las estrategias de afrontamiento positivas, la aceptación y crecimiento personal influyeron positivamente en todas las dimensiones del bienestar psicológico, lo que sugiere que el aceptar la realidad de una situación estresante, puede dar apertura a que se considere al estresor como una oportunidad de crecimiento personal. Lo mismo ocurrió con la estrategia de búsqueda de apoyo social, que influyó positivamente en la autoaceptación, propósito de la vida y crecimiento personal. Este resultado confirma lo encontrado por Carillo et al. (2021), quienes encontraron que el apoyo social funciona como factor protector ante conductas de riesgo.

En lo que respecta a las estrategias negativas, el consumo de alcohol o drogas influye de manera negativa con el bienestar psicológico, en especial, con la autoaceptación, relaciones positivas y propósito de la vida. El consumo de alcohol o drogas afecta de manera más fuerte a las relaciones positivas. Esto sugiere que, a mayor consumo, las relaciones personales son menos estrechas y de confianza, la persona tiende aislarse, a sentirse frustrado y no está dispuesto a hacer concesiones. Debido a las medidas de distanciamiento físico, utilizar esta estrategia de afrontamiento es un factor de riesgo considerable para el bienestar, no solo en quienes usan la estrategia, sino también para las personas de su entorno. Una actitud pasiva ante las situaciones estresantes también es un predictor de un menor bienestar psicológico, lo que significa que refrenar el afrontamiento puede provocar que la persona no se sienta satisfecha consigo misma, perciba que no tiene la capacidad de mejorar su entorno, no se de cuenta de las oportunidades que le rodean y perciba que su vida no tiene ni sentido, ni propósito. Estos resultados coinciden con los encontrados por Zacher y Rudolph (2020) y MacIntyre et al. (2020), cuyos resultados también categorizaron las estrategias de afrontamiento en positivas y negativas según su relación con la satisfacción de la vida.
Este estudio encontró que en el contexto del COVID-19, se utilizan más estrategias que impactan negativamente el bienestar psicológico que estrategias positivas. Este patrón tiene implicaciones clínicas considerables. Las dos estrategias positivas que pueden amortiguar el efecto negativo de la pandemia en el bienestar psicológico son aceptación y búsqueda de apoyo social. La posibilidad de emplear estas dos estrategias es afectada por la pandemia del COVID-19. En República Dominicana, específicamente, la desconfianza en las autoridades y en el personal de salud (Mencía-Ripley et al., 2021) puede entorpecer los procesos de aceptación del estresor. En segundo lugar, las medidas de distanciamiento físico obstaculizan la posibilidad de buscar apoyo social para recibir empatía y asistencia para enfrentar el estresor. Considerando que las estrategias de afrontamiento que mayormente están siendo utilizadas en el contexto de pandemia predicen un bajo bienestar psicológico, y las que predicen bienestar no siempre se pueden emplear, la población queda sin estrategias de afrontamiento adecuadas a la situación, lo que aumenta el riesgo de desarrollar patologías clínicamente relevantes.

Este estudio tiene varias limitaciones que deben ser tomadas en cuenta para su interpretación. En primer lugar, ambos instrumentos de medición son de auto-reporte. Los instrumentos de auto-reporte pueden estar sesgados por la deseabilidad social, en la que los participantes contestan las preguntas de manera que cause mejor impresión. En segundo lugar, esta muestra de estudiantes universitarios está limitada a universidades privadas de Santo Domingo, y sus participantes son de posición socioeconómica media-alta y alta. En el futuro se recomienda contar con una muestra probabilística de los estudiantes del país, de forma que sea posible entender el rol de la posición socioeconómica en la relación entre estrategias de afrontamiento y bienestar psicológico. Es importante estudiar el rol de la personalidad en el efecto de las estrategias de afrontamiento sobre el bienestar psicológico. También, estudios futuros podrían explorar la flexibilidad psicológica en esta 
muestra, como mecanismo que permite decidir en qué momento y en qué contexto una estrategia cognitiva se emplea de manera efectiva (Dawson $\&$ Golijani-Moghaddam, 2020).

\section{Conclusiones}

La situación de pandemia ha afectado el bienestar y la salud mental de los jóvenes universitarios. Por esto, es necesario estudiar los mecanismos que pueden contrarrestar los efectos negativos que provocan las medidas de prevención del COVID19. En este estudio investigamos cuáles estrategias de afrontamiento predicen el bienestar psicológico en el contexto de pandemia, buscando proporcionar una mejor comprensión para ayudar a sobrellevar esta crisis que afecta el bienestar integral de los universitarios.

Los resultados encontrados resaltan indicadores positivos que predicen el bienestar psicológico en esta muestra, como son la aceptación y crecimiento personal y la búsqueda de apoyo social. Sin embargo, nuestros resultados tambien reflejan que los jovenes universitarios utilizan con más frecuencia estrategias de afrontamiento negativas que predicen un menor bienestar psicológico, como son el consumo de alcohol o drogas, refrenar el afrontamiento y la negación. Estos resultados apoyan parcialmente los hallazgos de Ahmad et al. (2021), quienes encontraron que las estrategias de afrontamiento positivas más utilizadas en el contexto de pandemia fueron las de aceptación, crecimiento personal, búsqueda de apoyo social y planeación; y las negativas, refrenar el afrontamiento, desconexión mental y centrarse en las emociones y desahogarse.

Considerando estos hallazgos y sus limitaciones, este estudio es relevante debido a sus implicaciones clínicas, ya que la protección sobre el bienestar que ofrecen las estrategias de afrontamiento positivas se pueden ver mitigadas por las medidas de confinamiento y aislamiento social de COVID-19. Esto refleja la necesidad de desarrollar planes de intervención que protejan la salud mental de las pobla- ciones afectadas por las consecuencias sociales, psicológicas y económicas que trajo la pandemia del COVID-19.

\section{Referencias}

Ahmad, S., Nasreen, L., Batool, S., \& Khalid, S. (2021). Family functioning and psychological well-being: The mediating role of coping strategies during COVID-19 lockdown in Pakistan. Polish Psychological Bulletin, 52(2), 162-171.

Anglim, J., \& Horwood, S. (2021). Effect of the COVID-19 pandemic and Big Five Personality on subjective and psychological wellbeing. Social Psychological and Personality Science, 194855062098304. https://doi.org/ 10.1177/1948550620983047

August, R. A., \& Dapkewicz, A. S. (2021). Benefit finding in the COVID-19 pandemic: College students' positive coping strategies. Journal of Positive School Psychology, 5(2), 73-86. https:// doi.org/10.47602/JPSP.V5I2.245

Blasco-Belled, A., Tejada-Gallardo, C., Torrelles-Nadal, C., \& Alsinet, C. (2020). The Costs of the COVID-19 on Subjective Well-Being: An Analysis of the Outbreak in Spain. Sustainability, 12(15), 6243. https://doi.org/10.3390/ su12156243

Brauner, J. M., Mindermann, S., Sharma, M., Johnston, D., Salvatier, J., Gavenčiak, T., Stephenson, A. B., Leech, G., Altman, G., Mikulik, V., Norman, A. J., Monrad, J. T., Besiroglu, T., Ge, H., Hartwick, M. A., Teh, Y. W., Chindelevitch, L., Gal, Y., \& Kulveit, J. (2020). Inferring the effectiveness of government interventions against COVID-19. Science, eabd9338. https://doi.org/10.1126/ science.abd 9338

Cao, W., Fang, Z., Hou, G., Han, M., Xu, X., Dong, J., \& Zheng, J. (2020). The psychological impact of the COVID-19 epidemic on college students in China. Psychiatry Research, 287, 112934. https://doi.org/10.1016/j. psychres.2020.112934 


\section{Estrategias de Afrontamiento: efectos en el bienestar psicológico de universitarios durante la pandemia del COVID-19}

Carillo, L., Escamilla, M. L., M., R. J., \& González, V. (2021). Conducta alimentaria y consumo de alcohol durante el distanciamiento social por COVID-19 en México: Un estudio exploratorio. Ciencia y Sociedad, 46(2), 7-30.

Carver, C. S. (1997). You want to measure coping but your protocol's too long: Consider the Brief COPE. International Journal of Behavioral Medicine, 4(1), 92-100.

Carver, C. S., Scheier, M. F., \& Weintraub, K. J. (1989). Assessing coping strategies: A theoretically based approach. Journal of Personality and Social Psychology, 56(2), 267-283. https:// doi.org/10.1037/0022-3514.56.2.267

Chen, B., Sun, J., \& Feng, Y. (2020). How have COVID-19 isolation policies affected young people's mental health? - Evidence from Chinese college students. Frontiers in Psycho$\log y, 11,1529$. https://doi.org/10.3389/fpsyg. 2020.01529

Clabaugh, A., Duque, J. F., \& Fields, L. J. (2021). Academic stress and emotional well-being in United States college students following onset of the COVID-19 pandemic. Frontiers in Psychology, 12, 628787. https://doi. org/10.3389/fpsyg.2021.628787

Daly, M., \& Robinson, E. (2020). Psychological distress and adaptation to the COVID-19 crisis in the United States. Journal of Psychiatric Research. https://doi.org/10.1016/j.jpsychires.2020.10.035

Dawson, D., \& Golijani-Moghaddam, N. (2020). COVID-19: Psychological flexibility, coping, mental health, and wellbeing in the UK during the pandemic. Journal of Contextual Behavioral Science, 17, 126-134. https://doi. org/10.1016/j.jcbs.2020.07.010

Díaz, D., Rodríguez-Carvajal, R., Blanco, A., Moreno-Jiménez, B., Gallardo, I., Valle, C., \& Van Dierendonck, D. (2006). Adaptación española de las escalas de bienestar psicológico de Ryff. Psicothema, 18(3), 572-577.
Dong, E., Du, H., \& Gardner, L. (2020). An interactive web-based dashboard to track COVID-19 in real time. The Lancet Infectious Diseases, 20(5), 533-534. https://doi.org/10.1016/S14 73-3099(20)30120-1

Fluharty, M., \& Fancourt, D. (2021). How have people been coping during the COVID-19 pandemic? Patterns and predictors of coping strategies amongst 26,016 UK adults. $B M C$ Psychology, 9(1), 1-12. https://doi.org/10.1186 Is40359-021-00603-9

Foa, R. B., Gilbert, S., \& Fabian, M. O. (2020). COVID-19 and subjective well-being: Separating the effects of lockdowns from the pandemic. AvailableatSSRN:https://ssrn.com/abstract=36740. https://ssrn.com/abstract=3674080

Folkman, S., \& Lazarus, R. S. (1980). An analysis of coping in a middle-aged community sample. Journal of Health and Social Behavior, 21(3), 219. https://doi.org/10.2307/2136617

Genç, E., \& Arslan, G. (2021). Optimism and dispositional hope to promote college students' subjective well-being in the context of the COVID-19 pandemic. Journal of Positive School Psychology, 5(2), 87-96. https://doi. org/10.47602/JPSP.V5I2.255

Gianni, S. (2020). COVID-19 y educación superior: De los efectos inmediatos al día después. Revista Latinoamericana de Educación Comparada, 11(17), 1-57. http://www.iesalc.unesco. org/wp-content/uploads/2020/04/COVID19-060420-ES-2.pdf

Gol, A. R., \& Cook, S. W. (2004). Exploring the underlying dimensions of coping: A concept mapping approach. Journal of Social and Clinical Psychology, 23(2), 155-171. https:// doi.org/10.1521/jscp.23.2.155.31021

Husky, M. M., Kovess-Masfety, V., \& Swendsen, J. D. (2020). Stress and anxiety among university students in France during Covid-19 mandatory confinement. Comprehensive Psychiatry, 102, 152191. https://doi.org/10.1016/j.com ppsych.2020.152191 
Hyland, P., Shevlin, M., McBride, O., Murphy, J., Karatzias, T., Bentall, R. P., Martinez, A., \& Vallières, F. (2020). Anxiety and depression in the Republic of Ireland during the COVID-19 pandemic. Acta Psychiatrica Scandinavica, 142(3), 249-256. https://doi.org/10.1111/ acps. 13219

Islam, A., Barna, S. D., Raihan, H., Khan, N. A., \& Hossain, M. T. (2020). Depression and anxiety among university students during the COVID-19 pandemic in Bangladesh: A web-based cross-sectional survey. PLoS ONE, 15(8), e0238162. https://doi.org/10.1371/ journal.pone. 0238162

Jacobs, R., Lanspa, M., Kane, M., \& Caballero, J. (2021). Predictors of emotional wellbeing in osteopathic medical students in a COVID-19 world. Behavioral Health, 121(5), 455-461. https://doi.org/10.1515/jom-2020-0272

Joshanloo, M. (2016). Revisiting the empirical distinction between hedonic and eudaimonic aspects of well-being using exploratory structural equation modeling. Journal of Happiness Studies, 17(5), 2023-2036. https://doi. org/10.1007/s10902-015-9683-z

Joshanloo, M. (2019). Investigating the relationships between subjective well-being and psychological well-being over two decades. Emotion, 19(1), 183-187. https://doi.org/10.1037/ emo0000414

Lahav, Y. (2020). Psychological Distress Related to COVID-19 - The Contribution of Continuous Traumatic Stress. Journal of Affective Disorders, 277, 129-137. https://doi.org/10. 1016/j.jad.2020.07.141

Litman, J. A. (2006). The COPE inventory: Dimensionality and relationships with approach- and avoidance-motives and positive and negative traits. Personality and Individual Differences, 41(2), 273-284. https://doi.org/10.1016/j. paid.2005.11.032

MacIntyre, P. D., Gregersen, T., \& Mercer, S. (2020). Language teachers' coping strategies during the COVID-19 conversion to online teaching: Correlations with stress, wellbeing and nega- tive emotions. System, 94, 102352. https:// doi.org/10.1016/j.system.2020.102352

Martínez-Ortega, Y., Gomà-i-Freixanet, M., \& Fornieles-Deu, A. (2018). Versión española del COPE-48: Evaluación del afrontamiento en pacientes psiquiátricos ambulatorios. Terapia Psicológica, 36(1), 9-14. https://scielo.conicyt. cl/scielo.php?script=sci_arttext $\&$ pid $=$ S071848082018000100013

Martínez-Ortega, Y., Gomà-i-Freixanet, M., \& Fornieles Deu, A. (2016). The COPE-48: An adapted version of the COPE inventory for use in clinical settings. Psychiatry Research, 246, 808-814. https://doi.org/10.1016/j.ps ychres.2016.10.031

Mencía-Ripley, A., Paulino-Ramírez, R., Féliz Matos, L., Ruiz-Matuk, C. B., \& Sánchez-Vincitore, L. V. (2021). Psychological responses to the COVID-19 outbreak are related to trust in public institutions: Implications for management of emerging infectious diseases. InterAmerican Journal of Medicine and Health, 4. https://doi.org/https://doi.org/10.31005/ iajmh.v4i.164

Ministerio de Salud Pública de la Republica Dominicana. (2021). Coronavirus - República Dominicana. https://coronavirusrd.gob.do/

Morote-Jayacc, P. V., Sandoval, K. D., Moreno-Molina, M., \&Taype-Rondan, Á. (2020). Estudios de salud mental en estudiantes de Medicina en el contexto de la COVID-19. Revista Colombiana de Psiquiatria, 49(4), 223-224. https:// doi.org/10.1016/j.rcp.2020.07.005

Noor, F. M., \& Islam, M. M. (2020). Prevalence and associated risk factors of mortality among COVID-19 patients: A Meta-analysis. Journal of Community Health, 45(6), 12701282. https://doi.org/10.1007/s10900-02000920-x

Patias, N. D., Von Hohendorff, J., Cozzer, A. J., Flores, P. A., \& Scorsolini-Comin, F. (2021). Mental health and coping strategies in undergraduate students during COVID-19 pandemic. Trends in Psychology, 29(3), 414-433. https://doi. org/10.1007/s43076-021-00069-z 


\section{Estrategias de Afrontamiento: efectos en el bienestar psicológico de universitarios durante la pandemia del COVID-19}

Rathe, M. (2020). Dominican Republic: The response to the COVID-19 pandemic in 2020. Gaceta Médica de Caracas, 128(2), 227-235. https:// doi.org/10.47307/GMC.2020.128.s2.10

Rodríguez-Hidalgo, A. J., Pantaleón, Y., Dios, I., \& Falla, D. (2020). Fear of COVID-19, stress, and anxiety in university undergraduate students: A predictive model for depression. Frontiers in Psychology, 11, 591797. https:// doi.org/10.3389/fpsyg.2020.591797

Rogowska, A. M., Kuśnierz, C., \& Bokszczanin, A. (2020). Examining anxiety, life satisfaction, general health, stress and coping styles during COVID-19 pandemic in Polish sample of university students. Psychology Research and Behavior Management, 13, 797-811. https:// doi.org/10.2147/prbm.s266511

Roth, S., \& Cohen, L. J. (1986). Approach, avoidance, and coping with stress. American Psychologist, 41(7), 813-819. https://doi. org/10.1037/0003-066X.41.7.813

Ryan, R. M., \& Deci, E. L. (2001). On happiness and human potentials: A review of research on hedonic and eudaimonic well-being. Annual Review of Psychology, 52, 141-166. https:// doi.org/10.1146/annurev.psych.52.1.141

Ryff, C. D. (1989). Happiness is everything, or is it? Explorations on the meaning of psychological well-being. Journal of Personality and Social Psychology, 57(6), 1069-1081. https:// doi.org/10.1037/0022-3514.57.6.1069

Ryff, C. D. (2014). Psychological well-being revisited: Advances in the science and practice of eudaimonia. Psychotherapy and Psychosomatics, 83(1), 10-28. https://doi.org/10.1159/ 000353263

Ryff, C. D., \& Singer, B. (1996). Psychological wellbeing: Meaning, measurement, and implications for psychotherapy research. Psychotherapy and Psychosomatics, 65(1), 14-23. https://doi. org/10.1159/000289026

Salari, N., Hosseinian-Far, A., Jalali, R., Vaisi-Raygani, A., Rasoulpoor, S., Mohammadi, M.,
Rasoulpoor, S., \& Khaledi-Paveh, B. (2020). Prevalence of stress, anxiety, depression among the general population during the COVID-19 pandemic: A systematic review and meta-analysis. Globalization and Health, 16, 57. https://doi.org/10.1186/s12992-02000589-w

Schiavi, M. C., Spina, V., \& Zullo, M. A. (2020). Love in the time of COVID-19: Sexual function and quality of life analysis during the social distancing measures in a group of Italian reproductive-age women. The Journal of Sexual Medicine, 17, 1407e1413.

Stanisławski, K. (2019). The Coping Circumplex Model: An Integrative Model of the Structure of Coping With Stress. Frontiers in Psychology, 10, 694. https://doi.org/10.3389/ fpsyg.2019.00694

Tran, B. X., Nguyen, H. T., Le, H. T., Latkin, C. A., Pham, H. Q., Vu, L. G., Le, X. T. T., Nguyen, T. T., Pham, Q. T., Ta, N. T. K., Nguyen, Q. T., Ho, C. S. H., \& Ho, R. C. M. (2020). Impact of COVID-19 on economic well-being and quality of life of the Vietnamese during the national social distancing. Frontiers in Psychology, 11, 565153. https://doi.org/10.3389/ fpsyg.2020.565153

Valladolid, V. C. (2021). The Role of Coping Strategies in the Resilience and Well-Being of College Students during COVID-19 Pandemic. Philippine Social Science Journal, 4(2), 30-42. https:// doi.org/10.52006/main.v4i2.342

Van Bavel, J. J., Baicker, K., Boggio, P. S., Capraro, V., Cichocka, A., Cikara, M., Crockett, M. J., Crum, A. J., Douglas, K. M., Druckman, J. N., Drury, J., Dube, O., Ellemers, N., Finkel, E. J., Fowler, J. H., Gelfand, M., Han, S., Haslam, S. A., Jetten, J., ... Willer, R. (2020). Using social and behavioural science to support COVID-19 pandemic response. Nature Human Behaviour, 4(5), 460-471. https://doi.org/10.1038/ s41562-020-0884-z 
van Dierendonck, D., Díaz, D., Rodríguez-Carvajal, R., Blanco, A., \& Moreno-Jiménez, B. (2008). Ryffs six-factor model of psychological well-being, a Spanish exploration. Social Indicators Research, 87(3), 473-479. https:// doi.org/10.1007/s11205-007-9174-7

Wang, X., Hegde, S., Son, C., Keller, B., Smith, A., \& Sasangohar, F. (2020). Investigating Mental Health of US College Students During the COVID-19 Pandemic: Cross-Sectional Survey Study. Journal of Medical Internet Research, 22(9), e22817. https://doi.org/10.2196/22817

Williamson, E. J., Walker, A. J., Bhaskaran, K., Bacon, S., Bates, C., Morton, C. E., Curtis, H. J., Mehrkar, A., Evans, D., Inglesby, P., Cockburn, J., McDonald, H. I., MacKenna, B., Tomlinson, L., Douglas, I. J., Rentsch, C. T., Mathur, R., Wong, A. Y. S., Grieve, R., ... Goldacre, B. (2020). Factors associated with COVID-19-related death using OpenSAFELY. Nature, 584(7821), 430-436. https:// doi.org/10.1038/s41586-020-2521-4

Wood, D., Crapnell, T., Lau, L., Bennett, A., Lotstein, D., Ferris, M., \& Kuo, A. (2018). Emerging adulthood as a critical stage in the life course. In Handbook of life course health development (pp. 123-143). Springer, Cham.
World Health Organization. (2020). Considerations in adjusting public health and social measures in the context of COVID-19. In World Health Organisation Interim Guidance (Issue November). https://www.who.int/ publications-detail/risk-

Wu, F., Zhao, S., Yu, B., Chen, Y.-M., Wang, W., Song, Z.-G., Hu, Y., Tao, Z.-W., Tian, J.-H., Pei, Y.-Y., Yuan, M.-L., Zhang, Y.-L., Dai, F.-H., Liu, Y., Wang, Q.-M., Zheng, J.-J., Xu, L., Holmes, E. C., \& Zhang, Y.-Z. (2020). A new coronavirus associated with human respiratory disease in China. Nature, 579, 265-269. https://doi.org/10.1038/s41586020-2008-3

Zacher, H., \& Rudolph, C. W. (2021). Individual differences and changes in subjective wellbeing during the early stages of the COVID-19 pandemic. American Psychologist, 76(1), 50-62. https://doi.org/10.1037/amp0000702 